\title{
THE NEUROKERATIN IN THE MEDULLARY SHEATHS OF THE PERIPHERAL NERVES OF MAMMALS.
}

By Shinkishi Hatai.

(From the Neurological Laboratory of the University of Chicago.) With Psate IV.

At as early a date as 1876 , Ewald and KëHNE announced that the nervous system contains a horn-like substance which has a great resistance to chemical reagents and especially to gastric and tryptic digestive fluids. On account of its similarity to the horny material found in other tissues, they termed the substance obtained from the nervous system "Neurokeratin." Josephine Chevaliek, in 1884 , demonstrated the presence of the neurokeratin in the medullary sheath of the peripheral nerve fibers, by means of chemical analysis. Later (1890) Chittenden again took up the problem of the neurokeratin with Professor KüHNE, the first investigator of it, and not only confirmed the previous statement of EWALD and KüHNE, but at the same time cleared up many obscure points. In addition to the chemical determination of it, they showed also that the neurokeratin network in the medullary sheath may be demonstrated under the microscope by treating the fiber with boiling alcohol, ether and digestive fluids. By examining under the microscope the slides thus prepared K̈̈HNE and CHITTENDEN arrived at the conclusion that there are two layers of the neurokeratin network in the medullary sheath; one closely surrounding the axis cylinder, the other lying immediately beneath the primitive sheath.

Through the investigation of these writers just mentioned, the new field of the study was opened for the neurologist. There are a large number of articles concerning the neuroker- 
atin, but it is not necessary to review them in detail. Generally speaking, two different opinions are held by the recent investigators; one, according to which the neurokeratin is not a peculiar substance, but an artefact produced by the reagents employed, an albuminous substance (RANviEk) or a nucleoproteid (WYNN). This view is held by several histologists, for instance, Engelmann, Gerlach, Kölliker and Laviowsky. The second view, that the neurokeratin is a peculiar substance in the nervous system and preexists as such, is held by most of the physiological chemists as well as by a number of histologists; for instance, Leyilg, Paiduino, Joseph, Schiffarerecker, KAPLAN and others.

In recent years the use of Weicert's and HeIDENinin's haematoxylin has given many opportunities to study the structure of the neurokeratin, and as a consequence, a large number of reports have already been made. Most of the recent investigators are inclined to believe in the preexistence of the neurokeratin in the medullary sheath, on both chemical and histological grounds, and moreover the complexity of the neurokeratin increases as the technique improves.

Before discussing the literature further, I shall give an account of my own results.

For this investigation, the nerve fibers were preserved in 10\% formalin and cut into sections ro/e thick after the usual paraffine technique. The sections were stained with the "bluc solution" $"$ and decolorized sufficiently with diluted $\mathrm{NH} \mathrm{H}_{\mathrm{OH}}$. In some cases the blue solution was used without subsequent decolorization.

The preparations thus treated show the double layers of the neurokeratin network in the medullary sheath, one layer closely surrounding the axis cylinder, while the other lies just beneath the primitive sheath as was stated by EwALD and $\mathrm{K} \ddot{\mathrm{i}} \mathrm{HNE}$ and others. Such an arrangement of the neurokeratin

\footnotetext{
1 Preparation of the "blte solution" and its application to the miroscopical sections is given in my other paper,-- "On the Nature of the Periccllular Net. work." Journ. Comp. Neural., Vol. XIII, No. 2, p. ItI.
} 
can best be seen by examining the cross-section of the nerve fibers.

Fig. I shows the cross-section of the peripheral nerve of the cat, treated by the method mentioned above. The neurokeratin stains an intense pink, while the rest of the structure remains for the most part unstained ; the axis cylinder presents, however, a taint blue tint. It may easily be seen that each fiber shows the double rings of the neurokeratin, one along the outer margin and the other along the inner margin of the medullary sheath. These rings, however, are not complete circles, but appear as broken lines or are composed of dots. The size as well as number of the dots in each ring is variable. In some cases, instead of minute dots, a continuous long line is noticed, and in still other cases filaments more or less tangential to the inner ring are observed. The latter appearance is more common in the rings which immediately surround the axis cylinder. In some cases, these two rings are connected with each other by means of threads of neurokeratin which run perpendicularly to the long axis of the fibers and in cross-sections give an appearance of radiations from the central ring; in other words, wheel-like structures are formed by means of connecting bands between the two rings. The manner of the distribution of the connecting band is so irregular that it is almost impossi ble to give a general description of all the different appearances. I can merely state that the number of the connecting bands just mentioned is not constant, sometimes six or seven being observed and in some cases more than a dozen. Furthermore, the spaces formed by the connecting bands are also variable in size and shape. Some of the connecting bands are united with others by meanz of the delicate side-branches which they give off. In some instances, three rings are noticed (Fig. I, $b$ ). The reason for this irregular distribution of the neurokeratin in the cross-section of the nerve fibers is easily understood if one examines the longitudinal sections of the same fibers, and to these we now turn.

The longitudinal view of the nerve fibers is shown in Figures 2, 3 and 4 . The preparations were made from the peri- 
pheral nerves of the dog, using the same technique as was employed for the cross-section of the fibers.

Fig. 2 shows the general arrangement of the nerve fibers, as well as the manner of distribution of the neurokeratin. In this figure, the well known cone-shaped or funnel-shaped structures are shown clearly. The cone, as is known already, is formed by the network of the neurokeratin; the basal or wide end attaches to the outer layer of the neurokeratin sheath, while the narrow tip is attached to the neurokeratin sheath which directly surrounds the axis cylinder. In many cases, instead of the single cone there is the appearance of two cones united by their apices and at their bases attached to the outer margin of the sheath, as is shown in Fig. 2. The shape, size, direction, as well as number in each internode of the nerve fiber, is not constant. In some fibers the distance between the two narrow tips of the cones is quite regular, and the median longitudinal section of such fiber shows it to be divided into equal segments by means of the neurokeratin funnels or cones. (Fig. 2, $f$ ). Both the outer and inner layers of neurokeratin network are continuous throughout the entire length of the nerve fiber, as they are not interrupted at the nodes of RANviER (Fig. 2, $a, g$.) The structures of the cones and their relation to the two sheathing layers are shown in Fig. 4. I have mentioned already that double rings of the neurokeratin are noticed in examining the cross-sections of the fibers, and further gave the hint that the dots forming the rings are connected by means of delicate filaments of the same substance, and these connecting filaments are in turn continuous by means of the side-branches. Such a complicated arrangement may be understood by examining the structures drawn in Fig. 2 and Fig. 4. Since the two sheathing layers of the neurokeratin are connected either by radial filaments from the inner sheath toward the outer layer or by the branches which are sent off from the inner surface of the cones toward the inner neurokeratin sheath, there is thus formed a complicated arrangement of the neurokeratin in the cross-sections of the fibers. In most cases, the outermost layer of the neurokeratin has fine meshes, as is 
shown in Fig. 2, $b$, while in some cases larger spaces of the network are noticed at the small end of the funnel (Fig. 3) while the basal portion of the funnel has much finer meshes.

WynN ('Oo), who studied the medullary sheath by using the PAL-Weigert technique, states that each cone is composed of six segments, placed at regular distances apart, and converging from the primitive sheath to the axis cylinder. The present writer has examined a large number of preparations stained with PAL-WeIgert technique, but fails to confirm this result, although, in some cases, there are six dots placed at regular distances (Fig. $\mathrm{I}, a$ ) and located in some cases along the inner margin of the primitive sheath and sometimes closely surrounding the axis cylinder. A careful examination, however, reveals that in most cases more than six dots are to be seen, and furthermore, each dot is not a single dot but composed of several irregular lines and minute dots, thus giving a structure such as is to be seen in my preparations. According to WLassak's ('OO) investigation, the PAL-Weigert technique stains only the substance known as cerebrin, which is a constituent of the myelin, but not of the neurokeratin. If this statement of WLassak is true, the PAL-Weigert technique shows a distribution of the cerebrin in the medullary sheath, but not the distribution of the neurokeratin. The six rods described by WYNN, therefore, may prove that the cerebrin in the medullary sheath is distributed in a special relation to the cones; in other words, the cerebrin and neurokeratin are closely interdependent in their distribution. Wyns arrived, however, at the conclusion that the substance which forms the cones is neither a fatty body, nor neurokeratin, but a nucleoproteid. The statement given by WYNN, that the six dots given in the medullary sheath brought out by PAL-WEIGERT technique, are not the neurokeratin, seems very probable, for by comparing sections by the PAL-WeIGERT method with mine for neurokeratin, the dots of the former are seen to be of a size comparable with the spaces formed by the neurokeratin net in my slides and thus the two preparations give complementary pictures (Fig. 5). Passing by the contradictory statements of WynN 
and WLASSAK, it is only necessary for me to accept the statements that the cones described by Wisx are not true neurokeratin but a substance (either neucleoproteid or cerebrin) precipitated along the walls of funnels, and as a consequence, to conclude that WYNN is dealing with some substance other than neurokeratin. As a consequence, WyNn failed to obtain the network structure of the neurokeratin. The results obtained by the PAL-WeIgerT technique are, however, extremely interesting, for the preparations stained by this method reveal the manner of distribution of the cerebrin (WLASSAK) which is complementary to that of the neurokeratin and thus indicate indirectly the distribution of the neurokeratin.

The structure of the medullary stheath of the peripheral nerves, as revealed by these sections, seems to be as follows:

The neurokeratin network which contains the myelin consists, first, of two thin layers, one beneath the prinitive sheath, the other around the axis cylinder; these two layers are, however, continuous at the nodes of RANVIER; second, of a chain of cones connected with both layers, the base of a cone being attached to the peripheral layer, the apex in the inner layer, each cone being made up of a continuous band of neurokeratin exhibiting meshes of variable size and converging from the primitive sheath to the axis cylinder. The clefts of SchmintLANTERMANN occur at the situation of the cones, but in some cases they are also visible between the two cones. Furthermore, the cones present a great variability in number, size, and direction. These phenomena, just mentioned, may indicate that the Schmidt-Lanrermaniv clefts are produced artificially by rupture of the coagulated protoplasm along the walls of the cones. My own observations described above may advantageously be summarized as follows:

(I) The peripheral nerve-sheath contains two layers of the neurokeratin, one beneath the primitive sheath and the other along the axis cylinder. These two layers are connected by bands of neurokeratin which run obliquely from the outer to the inner layer, thus presenting a funnel or a cone-shaped appearance. 
(2) The neurokeratin sheath has a large number of pores or meshes and presents a net-like appearance. The size and shape of the meshes are, however, highly variable.

(3) Neither the outer nor inner layers of the neurokeratin are interrupted at the node of RANviER, but become continuous through it with the corresponding layers of the adjoining internodes.

(4) The present observations do not agree with Wynn's, but confirm the previous statements of EWALD and K̈̈HNE.

(5) The present technique brings out a more detailed structure of the neurokeratin than has been obtained by other investigators.

\section{BIBL.IOGRAPHY .}

EWALD and K ÜHNE: Ueber einen neuen Bestandtheil des Nervensystem. Virhand. d. naturh. med. Vireins zu Heidellerg. Vol. I, Heft 5, 1876.

Chevitifa, Jusfipine: Chemische Untersuchung der Nervensubstanz. Zeitsch. f. I'hysiol. Chem., Bd. X, ז884.

Kthse and Curtrexues: On Neurokeratin. The Newe York Medical Journal, Feb. 22 and March 1, also in Zeit. f. Biol., Bd. 26, 189o.

KöI.1.ıkeR: I Iandbuch der Gewebelehre. Bd. II, 1896.

Whassak: Herkunft der Myelin. Arch. f. Entzercklungsmechanik der Organismen. Vol. VI, Heft IV, 1898.

Wysx, W. H.: The minute structure of the medullary sheath of nervefibers. Journ. Anat. Physiol., Vol. 34, 1000.

Kaplax, L.: Nervenfărbungen (Neurokeratin, Markscheide, Axencylinder). Arch.f. Psych. u. Nervenheidlkunde, Bd. 35, IIeft 3, 1900.

The above bibliography is supplementary to the references given by KörLIKER, IVYNi and KAYLAN. 
The following figures are free-hand drawings, using Zeiss Oc. 4 × obj. I-12.

Fig. r.-Cross-sections of the ventral root nerves of the cat, showing distribution of neurokeratin.

Fig. 2.-I Iongitudinal section of the ventral root nerves of the dog, showing form of cones.

Fig. 3.-Largely magnified drawing of a single nerve-fiber taken from the preparation used for Fig. 2, showing the meshwork in the cones.

Fig. 4.-Same a Fig. 3.

Fig. 5.--Enlarged view of a cross-section of a single nerve-fiber taken from the preparation used for Fig. I, showing neurokeratin framework.

Fig. 6. Cross-section of ventral root nerve of human spinal cord. Pai. WeIGER. Largely magnified. Showing masses of medullary substance in. cluded in neurokeratin framework. 

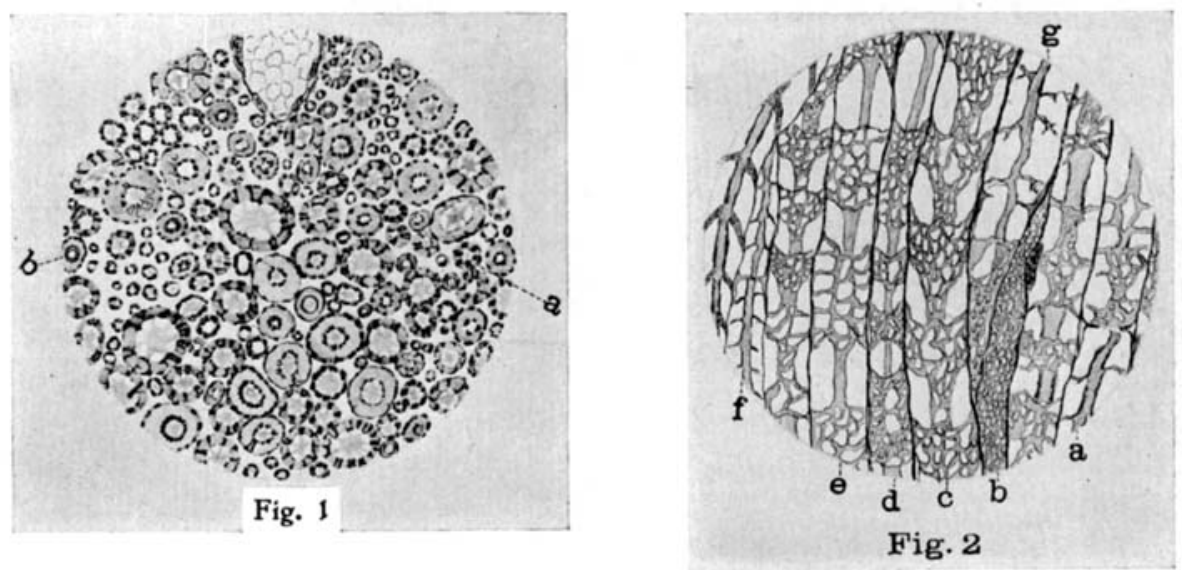

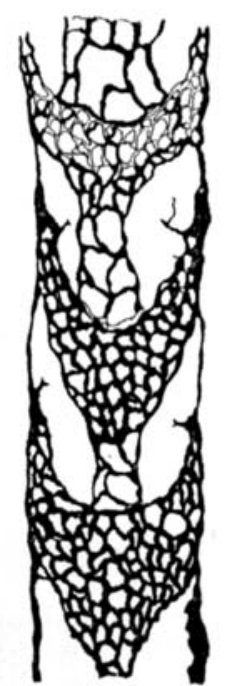

Fig. 3

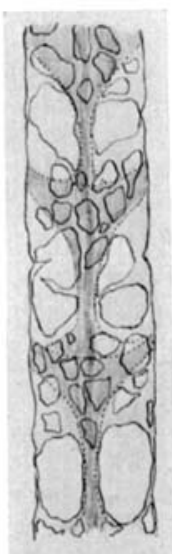

Fig. 4
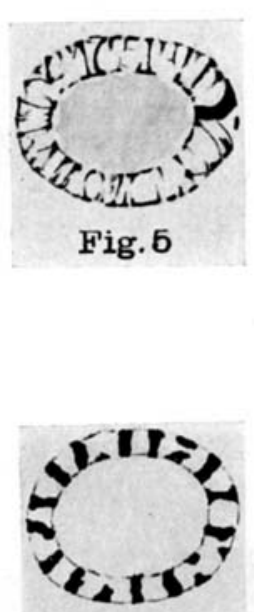

Fig. 6 